\title{
Prevalence of Temporomandibular Joint Dysfunction in Various Dental Patients According to the Different Types of Dental Treatments
}

\author{
Namrata Nitin Shinde ${ }^{1}$, Rutuja Balasaheb Shinde ${ }^{2}$, Poonam Hanmantrao Patil ${ }^{3}$ \\ 1, 2, 3 Department of Physiotherapy, Krishna Institute of Medical Sciences \\ (Deemed to Be University), Karad, Maharashtra, India.
}

\section{ABSTRACT}

\section{BACKGROUND}

Temporomandibular joint (TMJ) is mostly prone to various dysfunctions or abnormalities, even with a slight alteration in its normal function. Dental treatments in particular are known to cause stresses on this joint causing complications such as pain, tenderness, restricted movement, clicking sound etc. It can become severe with time and interfere in the daily living activities and affect the quality of life (QOL). Hence, this study was done to focus on a few common dental conditions to determine their effects on TMJ. The objective of this study was to evaluate the prevalence of TMJ dysfunction in dental patients with varying treatment durations.

\section{METHODS}

A total of 130 male and female dental patients who underwent /were undergoing various dental treatments were included, on the basis of age (18 - 45). The total number of samples were collected from 6 categories i.e., endodontic, orthodontic, cleaning / scaling, tooth extraction, filling, multiple treatments. FONSECA'S questionnaire was used as an outcome measure to determine the TMJ dysfunction.

\section{RESULTS}

Based on the assessment of all the 6 categories, major dysfunction was seen in the patients who underwent multiple treatments ( $86 \%$ ) followed by orthodontic (75\%), endodontic (73\%), tooth extraction (70\%), dental tooth filling (65\%) and the least was seen in patients of scaling / cleaning (61\%).

\section{CONCLUSIONS}

Prevalence of TMJ dysfunction has shown significant positive results in dental patients among which $71.5 \%$ of them have shown mild to severe TMJ dysfunction and $29.5 \%$ have shown negative results.

\section{KEY WORDS}

TMJ Dysfunction, FONSECA's Questionnaire, Orthodontic, Endodontic, Cleaning, Scaling, Tooth Extraction
Corresponding Author: Dr. Namrata Nitin Shinde, Department of Physiotherapy, Krishna Institute of Medical Sciences Deemed to Be University, Malkapur, Karad, Maharashtra, India. E-mail: namratashinde118@gmail.com

DOI: 10.14260/jemds/2021/423

How to Cite This Article:

Shinde NN, Shinde RB, Patil PH. Prevalence of temporomandibular joint dysfunction in various dental patients according to the different types of dental treatments. J Evolution Med Dent Sci 2021;10(28):20662071, DOI: $10.14260 /$ jemds/2021/423

Submission 06-03-2021,

Peer Review 11-05-2021

Acceptance 18-05-2021,

Published 12-07-2021.

Copyright (C) 2021 Namrata Nitin Shinde et al. This is an open access article distributed under Creative Commons Attribution License [Attribution 4.0 International (CC BY 4.0)] 


\section{BACKGROUND}

Temporomandibular joint (TMJ) is a synovial joint between the temporal bone of the skull above and the mandible below. It is essential in daily-living activities, i.e. chewing, speech, communication, and emotional expressions. ${ }^{1}$ TMJ is very prone to various dysfunctions or abnormalities after any alteration in its normal function. The normal working of joint is disturbed by several factors like mechanical, occupational and habits. ${ }^{2}$ The TMJ is unique within the body both structurally and functionally. Structurally it has 3 articular surfaces, which are articular tubercle, anterior part of mandibular fossa, and posterior non articular part formed by tympanic plate. Ligaments and muscles play a major role in the stability and mobility of the joint.

Temporomandibular disorder (TMD) is the most common pathological condition which is grouped under orofacial disorders, and the patient complains of pain, fatigue \& tenderness in the cranio-cervical muscles, limitation of mandibular movements and mandibular clicking. ${ }^{3}$ In relation to TMJ, in dental there are various treatments for which the patient has to keep his mouth open for prolonged time and has to come for several follow up visits for the dental procedure. Usually after such prolonged treatments, patient may suffer from TMJ conditions. ${ }^{4}$ Problems related to TMJ and population affected varies in different proportion. Three main categories are:

1. Myofascial pain dysfunction syndrome which involves tenderness or pain in the muscles that controls jaw function.

2. Internal derangement of the TMJ

3. Arthritis ${ }^{5}$

TMJ problems are frequently occurring with a prevalence rate of $45-70 \%$ in general population with varying signs, 30 $\%$ being aware of it, but only $3-12 \%$ people seek treatment for these complaints. As many as $10 \%$ of patients face severe problems in later stages of life.5,6 One study showed the long term effects of orthodontic treatment on periodontal health, comparing patients with untreated deranged teeth from those treated previously, suggested the difference was not significant. ${ }^{7}$ This infers as even a slight disturbance in the structural integrity of the TMJ with or without dental interference could lead to dysfunction. In another study focusing on the effect of lengthy root canal treatment on TMJ, showed $40-60 \%$ of population having considerable signs of TMD. ${ }^{8}$ Almost $60 \%$ of population showed positive response to TMJ dysfunction signs and symptoms after tooth extraction. ${ }^{9}$ As per the dentists opinion, patients with TMD tend to report that their pain was intensified by events such as stress, clenching, and eating. ${ }^{10}$ Likewise a number of studies have been done on TMJ with variable treatments like root canal treatment, bone grafting, dental implant, cosmetic smile designing, filling, cleaning, scaling, tooth extraction and so on have shown mixed results. Hence, in this study, a few common dental conditions were considered from the above list to determine their effects on TMJ respectively.

Examination of TMJ dysfunction can be done by using a questionnaire (Fonseca questionnaire) which includes some questions about the patients' various complaints. e.g., difficulty in movement, incomplete mouth opening, clicking sound during the movement, popping sounds, pain, decreased range of motion, locking of the jaw, difficulty in functional activities i.e., clenching, bruxism etc. after the final scoring, patient will be categorized accordingly. If the patient is suffering from TMD or not and if yes then whether it is mild, moderate, or severe dysfunction. ${ }^{3}$

\section{METHODS}

It is an observational analytical study that was carried out in Krishna hospital, Karad from November 2019 to April 2020. Research study was approved by the ethical committee of the institute (ethical no. 0146). Informed consent was taken from dental patients who had undergone treatment. i.e., orthodontic, endodontic, tooth extraction, cleaning / scaling, filling and also who underwent multiple treatments. The consent form was taken from willing participants and information regarding study had been stated. Samples were selected by the simple random sampling method (unrestricted). The sample size was calculated by using the formula $\mathrm{N}=4 \mathrm{pq} / \mathrm{I}^{2}$. where the value of $\mathrm{P}=80, \mathrm{q}=20$ and $\mathrm{l}=$ 7. The values of $p$ and $q$ were taken by the reference of related study $^{5}$ and by the experience of dentist. Sample size of 130 patients among which, 6 dental treatment groups that attended dental clinic for treatment were examined with Fonseca's questionnaire. ${ }^{3}$ The volunteers were informed to answer the questions as yes, no, and sometimes and that only one answer was to be marked for each question.

This included detail examination of clicking, crepitation, limitation, and pain and classified the signs and symptoms as mild, moderate, severe. ${ }^{3}$ All the subjects included in the study were based on inclusion criteria which included male and female patients in the age group of $18-45$ yrs. ${ }^{11}$ Patients who had undergone surgical procedures were excluded as a part of exclusion criteria. The detailed demographic data of the patient was also taken along with the consent form. On the basis of this detailed assessment further statistical analysis was taken.

\section{Procedure}

The study intended to determine the prevalence rate of temporomandibular dysfunction among dental patients and compared its degree within a few common dental procedures.

FONSECA questionnaire is a self-reported questionnaire used as an outcome measure in this study. The questions were based on the various symptoms of TMJ dysfunction i.e., pain, clicking sound, reduced range of motion, headache, difficulty in opening the mouth, pain on the nape or stiff neck, earaches, pain at the cranio-mandibular joint, clenching or grinding of teeth etc. answers to these questions were taken in the form of yes, no, and sometimes each marked as 0,5 , and 10 respectively.

The total score was calculated out of 100 and graded according to the scoring system based on subclassifications 0 to 15,20 to 40,45 to 60 and 70 to 100 . The individuals scoring 0 to 15 were considered to have No TMD, while 20 to 40 may 
have mild TMD. Individuals scoring between 45 and 60 were graded to have moderate TMD and finally a score of 75-100 was inferred as severe TMD. ${ }^{3}$ The result of the study was done based on detailed assessment.

\section{Statistical Analysis}

The study was concluded by statistical analysis of given outcome measure (by scoring of Fonsecaea questionnaire ${ }^{3}$ ). Statistics were done manually and by using statistic software InStat to verify the derived results. Percentage of all the groups were measured. The arithmetic mean and standard deviation was calculated for all the groups. The comparison between all the 6 groups was derived with the use of one-way analysis of variance i.e. ANOVA test, where the $P$ value was 0.0006 which is $<0.0001$, which means the difference was considered extremely significant.

\section{RESULTS}

Interpretation - Pie chart demonstrating the percentages of patients representing presence or absence of TMJ dysfunction. Approximately $29 \%$ of people have shown negative response and $72 \%$ of people have shown positive response.

Interpretation - Figure 1 shows that the highest prevalence of TMJ dysfunction is in patients undergoing multiple treatments (86\%) which is followed by orthodontic treatment (75\%), endodontic (73\%) and lastly tooth extraction (70\%). Other treatment procedures like dental tooth filling, cleaning / scaling have a lesser prevalence.
Likewise, individual result of each group is mentioned below:

1. Endodontic - From the total number of participants included in this group, $27 \%$ of participants have shown no TMD, $59 \%$ of them have shown mild TMD which was relatively high among the procedures selected, $10 \%$ of them have shown moderate TMD and only $4 \%$ of them have shown severe TMD.

2. Orthodontic -This is one of the procedures which requires longer treatment hours and is a relatively common procedure. About $75 \%$ of the participants undergoing this treatment reported some level of TMD, from the total number of participants included in this group, $45 \%$ of them have shown mild TMD, $22 \%$ of them have moderate TMD while $8 \%$ of the total participants suffered from severe TMD. No TMD was seen in $25 \%$ of these participants.

3. Cleaning / scaling - These are out patient procedures with short treatment duration. No participant undergoing this procedure showed any sign of severe dysfunction. Though, $61 \%$ of participants had mild and moderate dysfunction, $39 \%$ of participants did not have any TMD.

4. Tooth extraction - In this group, $30 \%$ of participants had shown no TMD, $55 \%$ of them had shown mild TMD, $7 \%$ of them had shown moderate TMD and $8 \%$ of them had shown severe TMD.

5. Tooth filling -Though no participants of this group suffered from severe dysfunction, about $53 \%$ had mild dysfunction and $12 \%$ had shown moderate dysfunction. From the total number of participants included in this group, $35 \%$ of participants had shown no TMD.

6. Multiple treatments - This is the group with highest prevalence of TMD, with $10 \%$ showing severe grade of dysfunction, as many as $38 \%$ showing mild and moderate dysfunction each and only $14 \%$ of participants suffered no TMD.

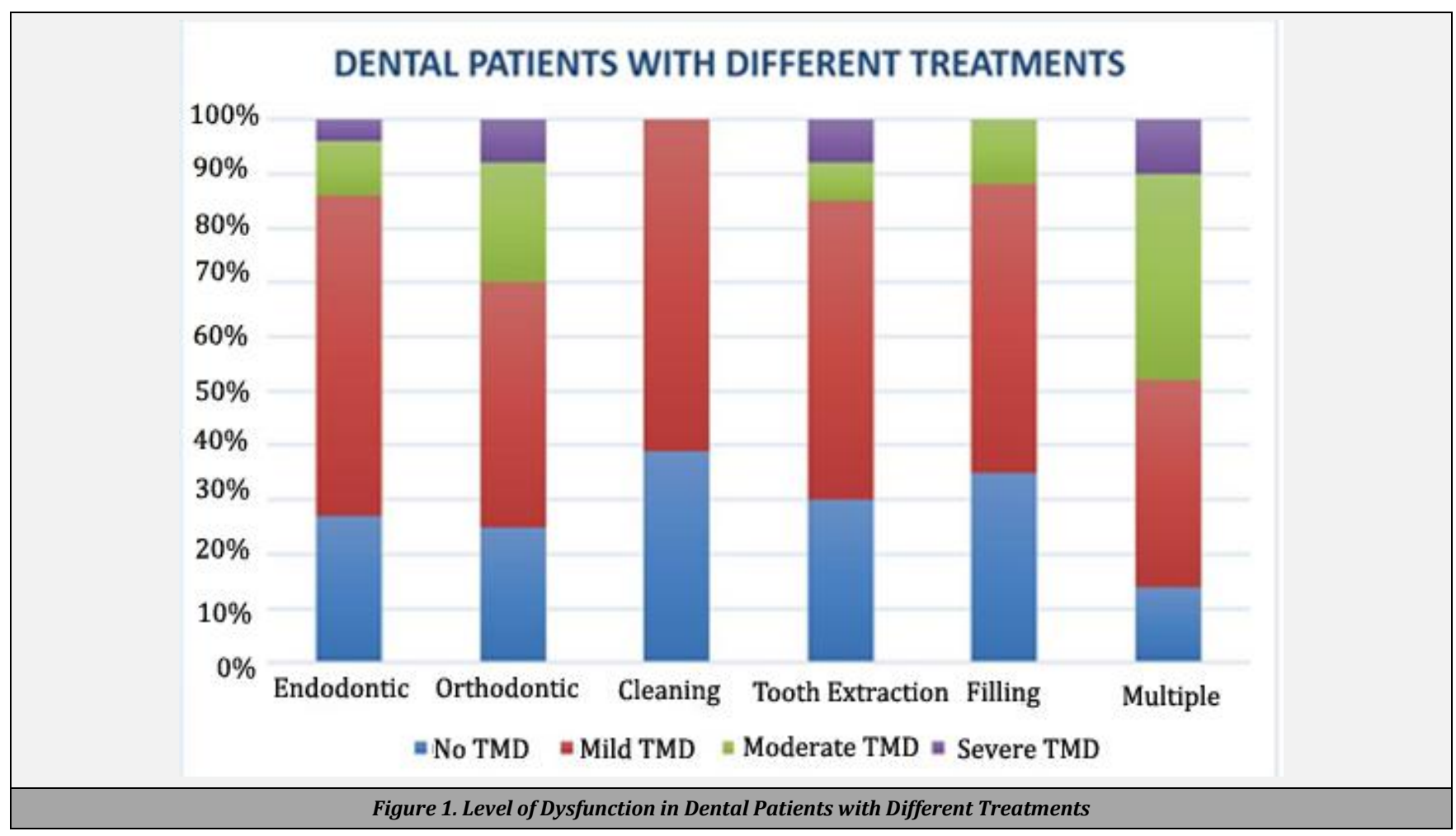



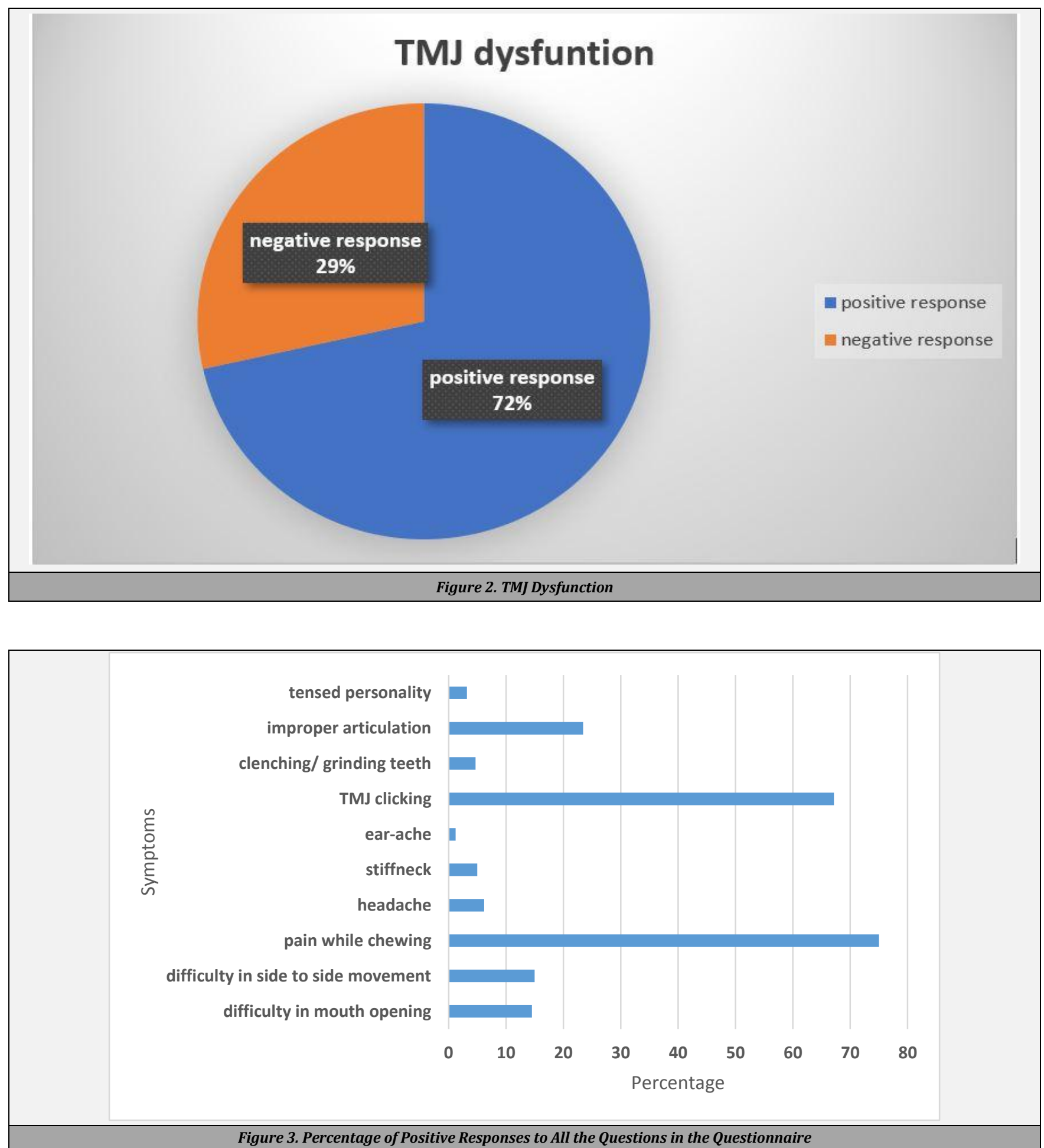

\begin{tabular}{|ccc|}
\hline Name of Treatment & Mean \pm Standard deviation & \% of TMD \\
Endodontic & $23.09 \pm 12.19$ & 73 \\
Orthodontic & $27.38 \pm 17.22$ & 75 \\
Cleaning & $20.00 \pm 7.07$ & 61 \\
Tooth extraction & $29.04 \pm 17.00$ & 70 \\
Filling & $26.42 \pm 12.95$ & 65 \\
Multiple treatment & $40.95 \pm 20.71$ & 86 \\
\hline Table 1. Mean, SD, and Percentage of TMD of Each Dental Treatment \\
\hline
\end{tabular}

\section{DISCUSSION}

The study has been conducted to determine the prevalence of TMJ dysfunction in dental patients. FONSECA questionnaire was used to assess the signs and symptoms ranging from mild to severe TMD. Responses of 128 participants were analysed out of which $73 \%$ were males and $27 \%$ were females between age 18 and 45 years. However, the participants included were sufficiently cooperative.

Approximately, $71.5 \%$ of the patients had shown positive response with temporomandibular joint dysfunction among all the patients with varying conditions. After analysing the data, the most affected groups were multiple treatment, orthodontic treatment, endodontic followed by tooth extraction treatment. The percentages of these procedures were as follows - $86 \%, 75 \%, 73 \%$ and $70 \%$ respectively. The least affected groups were tooth filling followed by cleaning / 
scaling with the percentage of $65 \%$ and $61 \%$ respectively. It could be observed through these results that a high prevalence of TMD was seen in all the dental procedures. Further, analysing the data using ANOVA test to establish a correlation between TMD and the common dental procedures, the $P$ value obtained was 0.0006 . These results indicate the presence of a positive co-relation of TMD among patients of dental procedures who are prone to TMD.

Similar results were obtained in the majority of preceding studies such as Cyril sadowsky et al. (1980), virginie tuerlings et al.(2004), k. k alshaban et al.(2018).2,6,12 The results of the present study are lined with Olsson and Lindquist (1992) where, muscular pain and tenderness were found to have greater positive response that is $75 \%$, among which mild TMD were present in $45 \%$ and moderate symptoms were shown in around $28 \%$ of them, whereas $2 \%$ showed severe symptoms. Concerning the prevalence of clicking sound in the present participants, the results of this study are similar to those Williamson's (1977) and Kelling et al. (1994), Yu-meng deng (1995), who found that $67 \%$ of the people from the total population had TMJ clicking. Teeth articulation was found to be one of the significant problems in post dental procedure with a prevalence rate of $23.45 \%$. One of the studies (virginie tuerlings, 2004) revealed that there could be a correlation between muscular tenderness, clicking and improper articulation.12,13 Affection of the mandibular range of motion was one of the major TMD findings among post dental patients with $15 \%$ prevalence in side to side range and $14.5 \%$ in elevation depression ranges. While the clinical examination of these patients, was difficult to achieve total objectivity, especially for the questions like clenching, grinding of teeth and considering themselves as a tensed / nervous person which involves a subjective response. Likewise, nearly identical results were obtained for the following questions that is stiffness of neck ( $6.2 \%)$ and headache (5.1\%). Whereas earache was very rare in this population. There has been much support for the concept that the duration of the treatment and the multiple sessions involved in the dental treatment can significantly upraise signs and symptoms of TMD. If these symptoms remain untreated it could affect quality of life (QOL) and can trigger more signs and symptoms with pain. Thus, more attention has to be given to this condition in order to avoid the adverse effects of it. Hence, it is recommended to have multiple and short treatment sessions where the patient does not have to keep the mouth open for long time and is allowed to take short breaks, thus preventing extra stress on temporomandibular joint and structures associated with it. Rendell JK, Norton LA et al. suggested that the interdisciplinary approach between dentist and physiotherapist helps in early diagnosis and improves the condition of TMJ. ${ }^{14}$

\section{CONCLUSIONS}

Research concluded that the temporomandibular joint dysfunction is very common among the dental patients irrespective of the type of treatment. The statistical analysis confirmed that the temporomandibular joint dysfunction was significant in both the genders. Among all the participants, male population have shown greater positive results than the female population.
Patients showing mild to moderate symptoms were highest in proportion than the severe ones. This study correlates with the earlier studies and their findings which says the longer treatment procedures lead to TMJ dysfunction. Additionally, it has been concluded that short dental treatments can also end up with having some level of TMJ dysfunction. So the study concludes that the main aetiology of TMD is long term opening of the mouth during dental procedures and several follow ups causing the stress on TMJ.

\section{Limitations}

1. There were less males in the study, so poor gender discrimination.

2. The study group size was small; hence more treatment protocols can be studied with larger population.

3. Age groups were mostly under 30 years of age.

Data sharing statement provided by the authors is available with the full text of this article at jemds.com.

Financial or other competing interests: None.

Disclosure forms provided by the authors are available with the full text of this article at jemds.com.

\section{REFERENCES}

[1] Ingawale $S$, Goswami T. Temporomandibular joint: disorders, treatments and biomechanics. Ann Biomed Eng 2009;37(5):976-96.

[2] AlShaban KK, Waheed ZGA. Prevalence of TMJ disorders among the patients attending the dental clinic of Ajman University of Science and Technology-Fujairah Campus, UAE. Int J Dent 2018;2018:9861623.

[3] Nomura K, Vitti M, Oliveira AS, et al. Use of the Fonseca's questionnaire to assess the prevalence and severity of temporomandibular disorders in Brazilian dental undergraduates. Braz Dent J 2007;18(2):163-7.

[4] Siddiqui TM, Wali A, Siddiqui F, et al. Effect of prolonged endodontic treatment on temporomandibular joint and masticatory muscles. World J Dent 2018;9(1):38-42.

[5] Mesnay W. Current role of orthodontists in treating temporo-mandibular disorders. J Dentofacial Anom Orthod 2012;15(2).

[6] Sönmez H, Sari S, Oray GO, et al. Prevalence of temporomandibular dysfunction in Turkish children with mixed and permanent dentition. J Oral Rehabil 2001;28(3):280-5.

[7] Sadowsky C, BeGole EA. Long-term status of temporomandibular joint function and functional occlusion after orthodontic treatment. Am J Orthod 1980;78(2):201-12.

[8] Sahebi S, Moazami F, Afsa M, et al. Effect of lengthy root canal therapy sessions on temporomandibular joint and masticatory muscles. J Dent Res Dent Clin Dent Prospects 2010;4(3):95-7.

[9] Dolatabadi MA, Lassemi E. Trauma to the temporomandibular joint following tooth extraction via dental students. Trauma Mon $2011 ; 16(4): 205$. 
[10] Wright EF, North SL. Management and treatment of temporomandibular disorders: a clinical perspective. J Man Manip Ther 2009;17(4):247-54.

[11] Greene CS, Laskin DM. Long-term status of TMJ clicking in patients with myofascial pain and dysfunction. J Am Dent Assoc 1988;117(3):461-5.

[12] Tuerlings V, Limme M. The prevalence of temporomandibular joint dysfunction in the mixed dentition. Eur J Orthod 2004;26(3):311-20.
[13] Deng YM, Fu MK, Hägg U. Prevalence of temporomandibular joint dysfunction (TMJD) in Chinese children and adolescents. A cross-sectional epidemiological study. Eur J Orthod 1995;17(4):305-9.

[14] De Toledo EG Jr, Silva DP, De Toledo JA, et al. The interrelationship between dentistry and physiotherapy in the treatment of temporomandibular disorders. J Contemp Dent Pract 2012;13(5):579-83. 